\title{
La lutte par piégeage contre Glossina fuscipes fuscipes pour la protection de l'élevage en République centrafricaine. IV. Impact entomologique, parasitologique et zootechnique
}

\author{
F. Le Gall ${ }^{1}$, F. Blanc ${ }^{2}$, J.P. Gouteux ${ }^{3}$, M. Mainguet ${ }^{4}$, D. Cuisance ${ }^{5}$, J.L. Lemesre ${ }^{6 *}$, S. \\ Nitcheman ${ }^{6}$, M. Cavaleyra ${ }^{6}$, F. D'Amicos ${ }^{5}$, E. Pounékrozou ${ }^{4}$, F. N'Dokoué ${ }^{4}$
}

\begin{abstract}
LE GALL (F.), BLANC (F.), GOUTEUX (J.P.), MAINGUET (M.), CUISANCE (D.), LEMESRE (J.L.), NITCHEMAN (S.), CAVALEYRA (M.), D'AMICO (F.), POUNÉKROUZOU (E.), N'DOKOUÉ (F.). La lutte par piégeage contre Glossina fuscipes fuscipes pour la protection de l'élevage en Rếpublique centrafricaine. IV. Impact entomologique, parasitologique et zootechnique. Revue Élev. Méd. vét. Pays trop., 1995, 48 (2) : 161-169

Le suivi d'un réseau d'élevage de 19 troupeaux zébus Mbororo a été mis en place pour évaluer l'impact d'une lutte par piégeage contre Glossina fuscipes fuscipes limitée aux abreuvoirs. Ce programme s'inscrit dans la stratégie d'une lutte intégrée contre les trypanosomoses bovines en République centrafricaine. Le piégeage permet d'abaisser les densités de $G$. f. fuscipes et de diminuer les prévalences trypanosomiennes. Ces effets se traduisent par l'amélioration des valeurs de l'hématocrite et par la diminution du nombre de traitements trypanocides réalisés. L'impact sur les productivités est plus difficile à mettre en évidence sur une courte période ; il apparaît cependant manifeste sur les paramètres calculés.
\end{abstract}

Mots clés : Bovin - Zébu - Trypanosomose - Glossina fuscipes fuscipes Lutte anti-insecte - Piège - Elevage - République centrafricaine.

\section{INTRODUCTION}

Le délestage pastoral du Sahel a renforcé le mouvement séculaire des éleveurs Mbororo en direction des savanes humides du Sud-Est, plus riches en pâturages mais infestées de glossines. Les trypanosomoses bovines sont ainsi l'un des problèmes majeurs des éleveurs Peul centrafricains, nécessitant l'utilisation massive de trypanocides. Cet élevage "sous la seringue" pose des problèmes techniques et économiques : coût des traitements, émergence de souches chimiorésistantes (21). L'Agence nationale de développement de l'élevage (ANDE) a donc développé une lutte antivectorielle contre Glossina fuscipes fuscipes, principal vecteur des trypanosomoses du bétail en République centrafricaine (RCA) $(6,7,8)$.

1. Banque mondiale, AGRTN, $1818 \mathrm{H}$ Street, N.W. Washington, DC 20433, Etats-Unis.

2. Fédération nationale des éleveurs centrafricains (FNEC), BP 1509, Bangui, République centrafricaine.

3. ORSTOM, s/c Université de Pau et des Pays de l'Adour, IPRA, $64000 \mathrm{Pau}$, France.

4. Agence nationale de développement de l'élevage (ANDE), BP 1509, Bangui, République centrafricaine.

5. CIRAD-EMVT, s/c Centre ORSTOM, BP 5045, 34032 Montpellier Cedex, France.

6. ORSTOM, Unité de Biologie parasitaire*, BP 5045, 34032 Montpellier Cedex, France.

Reçu le 2.9.1994, accepté le 27.7.1995.
Les essais de lutte entrepris en RCA contre $G$. $f$. fuscipes reposaient auparavant sur la pulvérisation d'un insecticide rémanant associée à l'isolement des galeries forestières traitées par des barrières de déboisement (27). Cette méthode de lutte visait l'éradication du vecteur. Elle ne donne de bons résultats qu'à court terme et sur une superficie limitée dont on peut assurer l'isolement par des barrières ; or la lutte en RCA doit être conduite à l'échelle du pays, applicable pour un cheptel important et doit tenir compte de la grande mobilité des éleveurs Peul. Le contrôle permanent des populations du vecteur constitue une alternative aux méthodes d'séradication». Ces techniques moins drastiques visent à contenir à un niveau suffisamment bas les prévalences trypanosomiennes du bétail, en abaissant les densités du vecteur et/ou en diminuant le contact bétail-vecteur. Ces pathologies ne constituent plus alors un problème sanitaire majeur et peuvent être contrôlées par un niveau acceptable de chimiothérapie $(6,8)$.

Une prise en charge technique et financière par les éleveurs de cette lutte contre $G$. $f$. fuscipes nécessitait la mise au point d'un outil bon marché, efficace contre ce vecteur et d'utilisation simple et individuelle. Le piège bipy' ramidal utilisé $(14,15)$ a été conçu dans cette optique.

Une évaluation de l'impact de la technique de piégeage était nécessaire préalablement à la diffusion-vulgarisation du piège (1) à grande échelle. Les impacts entomologique, parasitologique et zootechnique d'une lutte par piégeage limitée aux abreuvoirs fréquentés par le bétail sur un réseau de troupeaux suivis pendant une année; sont présentés dans cet article.

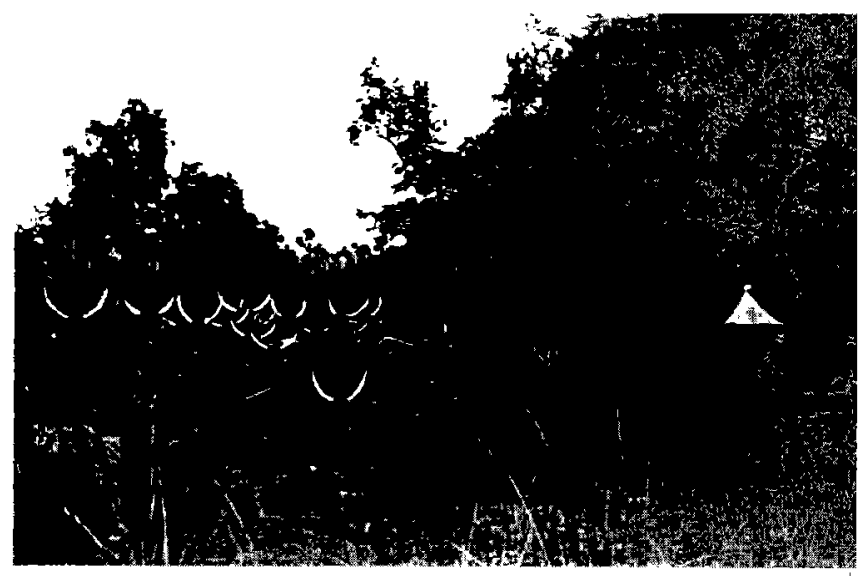

Figure 1: Piégeage d'un abreuvoir (cliché F. Le Gall). 
F. Le Gall F. Blanc J.P. Gouteux M. Mainguet D. Cuisance J.L. Lemesre S. Nitcheman M. Cavaleyra F. D'Amico E. Pounékrouzou F. N'Dokoué

\section{MATÉRIEL et MÉTHODES}

L'étude repose sur un suivi de troupeaux en conditions réelles. Cette approche permet de tenir compte des contraintes de terrain, difficilement reproductibles en milieu contrôlé, pour évaluer l'impact de la lutte et la faisabilité d'une prise en charge du piégeage par les éleveurs.

\section{Zone d'étude}

La région étudiée est celle d'Ouro-Djafoun dans la préfecture de la Ouaka, au centre-est de la RCA. Cette commune d'élevage a été créée en 1965 pour héberger les troupeaux des Mbororo venus du Cameroun ou de l'Ouest de la RCA. Ces éleveurs Peul pratiquent un élevage extensif de leur bétail zébu, avec une transhumance de saison sèche de novembre-décembre à avril-juin vers des zones plus au sud qu'ils atteignent après deux mois de déplacement itinérant et où ils séjournent environ trois mois. Ouro-Djafoun a une superficie d'environ $1500 \mathrm{~km}^{2}$ et est situé en zone soudano-guinéenne, avec une pluviométrie moyenne de 1 $500 \mathrm{~mm} / \mathrm{an}$ (source : station INRTV - Bambari, RCA). Les ligneux ont tendance à envahir la végétation graminéenne de ces savanes arbustives à Piliostigma thonningii, Annona senegalensis, Nauclea latifolia, Terminalia spp., Grewia spp. et Hymenocardia acida (24), traversées par de nombreuses galeries forestières.

Ouro-Djafoun se situe dans une aire géographique à forte pression trypanosomienne (19) : taux élevé de mortalités imputables aux trypanosomoses, coût important d'achat de trypanocides (560 F CFA dépensés par animal et par an, soit 3,3 traitements), densité glossinienne moyenne à forte (densité apparente par piège et par jour [DAP] moyenne sur la zone : 3 G. f. fuscipes). Trois espèces de glossines avaient été identifiées en 1963 sur cette commune : G. f. fuscipes, $G$. fusca congolensis et $G$. fuscipleuris (12). Une prospection entomologique préalable à la mise en place du réseau (2) a établi la rareté de $G$. fusca congolensis ( 6 spécimens récoltés pour $1746 \mathrm{G}$. $f$. fuscipes) et l'absence de G. fuscipleuris. G. $f$. fuscipes apparaît donc bien comme le vecteur de trypanosomoses dans cette commune; la lutte doit être dirigée contre cette espèce.

\section{Réseau d'élevage}

Le réseau comprend 19 troupeaux (initialement 673 animaux) : 17 troupeaux, comprenant 573 animaux au total, appartiennent à 13 éleveurs Mbororo ; 2 troupeaux de 50 têtes chacun ont été constitués par l'ANDE.

Les abreuvoirs de 12 troupeaux (436 animaux, lot P) sont protégés à l'aide de 1 à 4 pièges par abreuvoir selon la dimension de la galerie forestière. Les 7 troupeaux restant (237 animaux, lot NP) constituent le groupe témoin. Un élevage dispose de 2 abreuvoirs en moyenne. Le piégeage concerne les abreuvoirs de saison des pluies, puis les abreuvoirs de saison sèche sur les lieux de transhumance.
Le choix des élevages protégés et non protégés s'est fait de manière aléatoire. Aucune différence significative de DAP ni de prévalence trypanosomienne n'existe au départ entre les lots $P$ et NP. L'emplacement des campements de saison des pluies ainsi que l'effectif et le type de protection des troupeaux du réseau sont présentés sur la figure 2.

\section{Suivi du réseau}

La cohorte des 673 animaux a été suivie de juin 1990 à juin 1991 par une enquête prospective de type traité/témoin (ici piégé/non piégé). L'appréciation de la situation entomologique de la zone et l'identification des candidats potentiels permettent d'établir les conditions nécessaires à l'introduction d'un troupeau dans le réseau :

- présence de G. f. fuscipes aux abreuvoirs fréquentés par le troupeau (DAP supérieure ou égale à 1) ;

- acceptation par le propriétaire du marquage des animaux suivis et d'un suivi incluant des prises de sang sur ces animaux;

- assurance d'un retour au campement d'origine des troupeaux transhumants ;

- mode d'élevage, composition du troupeau, état zoosanitaire général sans spécificité nette par rapport aux troupeaux Mbororo de la région.

\section{Enquête}

Les visites trimestrielles ont été effectuées par l'équipe chargée du programme en juin 1990 (V1, début de saison des pluies), octobre 1990 (V2, fin de saison des pluies), février 1991 (V3, milieu de saison sèche) et juin 1991 (V4, début de saison des pluies). Les prélèvements sanguins ont été réalisés (jusqu'en février 1991) et les données zootechniques (jusqu'en juin 1991) ont été collectées lors de ces visites.

Trois agents responsables de la surveillance du réseau effectuaient bimensuellement en saison des pluies et mensuellement en saison sèche un piégeage des abreuvoirs des troupeaux témoins, l'inspection du dispositif de lutte et le relevé des captures des pièges de lutte (jusqu'en avril 1991), ainsi que le relevé des paramètres zootechniques.

\section{Paramètres recherchés et techniques utilisées}

\section{Entomologie}

L'évolution des densités glossiniennes aux abreuvoirs était suivie par un piégeage bimensuel (en saison des pluies) et mensuel (en saison sèche) de 2 à 4 jours à l'aide du piège bipyramidal Gouteux-ANDE (15). Les DAP étaient exprimées en glossines/piège/jour. 


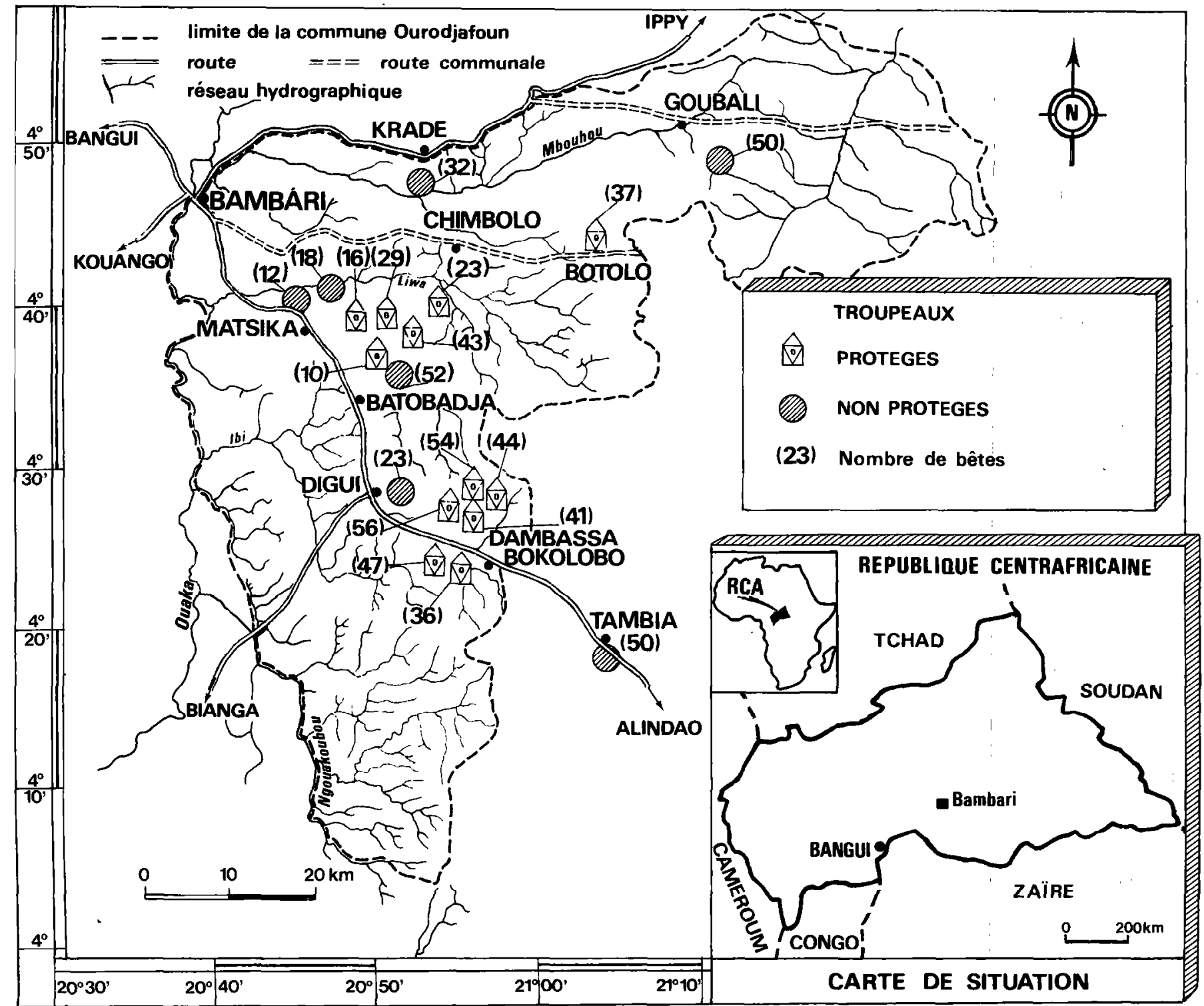

Figure 2 : Carte de situation du réseau Ouro-Djafoun.

\section{Parasitologie - Hématocrite}

Les trypanosomes étaient recherchés sur le bétail à partir de sang prélevé à la veine auriculaire en tube hématocrite, après une centrifugation de $5 \mathrm{~min}$ à 12000 tours $/ \mathrm{min}$ et lecture de l'hématocrite, à l'état frais après section de l'interface éléments figurés/sérum (26). Les lectures du frottis fixé et coloré au May-Grünwald Giemsa et de la goutte épaisse fixée et colorée au Giemsa ont été effectuées ultérieurement pour préciser l'espèce en cause.

`En janvier 1995, l'ILRAD et l'ILCA ont fusionné au sein de l'ILRI.

\section{Sérologie}

La recherche des antigènes trypanosomiens circulants sur le bétail était faite à partir de sang prélevé sur tube sec après aliquotage en cryotube et congélation. La technique ELISA était mise en œuvre au Laboratoire d'épidémiologie des grandes endémies tropicales (ORSTOM, Montpellier, France). Les sondes monoclonales spécifiques d'espèce (anticorps monoclonaux préparés in vitro à partir de formes procycliques de Trypanosoma b. brucei, $T$. congolense et $T$. vivax) ont été mises au point et gracieusement cédées par l'ILRAD* (25) qui n'a toutefois pas encore validé cette technique. 
F. Le Gall F. Blanc J.P. Gouteux M. Mainguet D. Cuisance J.L. Lemesre S. Nitcheman M. Cavaleyra F. D'Amico E. Pounékrouzou F. N'Dokoué

\section{Zootechnie}

Les animaux adultes étaient marqués au fer rouge à la corne et les jeunes à la croupe. Le nom, le sexe, l'âge, l'état zootechnique, le poids des veaux, le nombre de mises bas et les antécédents pathologiques étaient relevés au moment de la mise en place du suivi. Les données zootechniques suivantes étaient relevées au cours du suivi : l'état zootechnique (notation de 1 à 5 ), les mortalités, les ventes, les dons, le confiage, les naissances, le poids des veaux, la quantité de lait trait, le nombre de traitements réalisés contre les trypanosomoses, les événements pathologiques survenus entre chaque visite.

\section{Analyses des résultats}

Les analyses statistiques réalisées à l'aide du logiciel STAT-ITCF constituaient en un test de comparaison des moyennes (DAP), un test de comparaison des pourcentages (paramètres zootechniques), un test de MannWhitney (hématocrites et prévalences).

La réalisation d'un suivi en conditions réelles sur un nombre finalement restreint d'animaux entraînait certaines contraintes : l'amélioration de productivité due au piégeage allait être difficilement discernable au niveau du troupeau au bout d'une année, et risquait d'être masquée par l'ensemble des facteurs zoo-sanitaires non contrôlés influant sur la productivité du cheptel. La recherche de l'impact du piégeage sur la productivité s'est donc faite différemment d'une comparaison entre lots témoin et piégé pour les paramètres mortalité, fécondité et quantité de lait trait. Le calcul suivant a permis cette estimation : les productivités ont été mesurées d'une part sur l'ensemble des animaux détectés au moins une fois positif en parasitologie (ensemble $T+$ ) (109 animaux) et d'autre part sur l'ensemble des animaux restés négatifs au cours du suivi (ensemble T-) (564 animaux), indépendamment de leur appartenance aux lots $P$ et NP. Ces lots $P$ et NP ont ensuite été considérés comme composés d'animaux de ces ensembles $T+$ et $T$ - selon les prévalences observées au cours du suivi pour chaque lot $P$ ou NP. Si des différences de productivité entre animaux $T_{+}$ et animaux $T$-, et de prévalence entre lots $P$ et NP apparaissaient, cette péréquation permettait de les discerner.

\section{RÉSULTATS}

\section{Entomologie}

Le suivi des DAP de $G$. f. fuscipes aux abreuvoirs montre une régression significativement différente des DAP aux abreuvoirs piégés $(P)$ par rapport aux DAP des abreuvoirs non piégés (NP) au bout de deux mois en saison des pluies (fig. 3a) et d'un mois en saison sèche (fig. 3b,

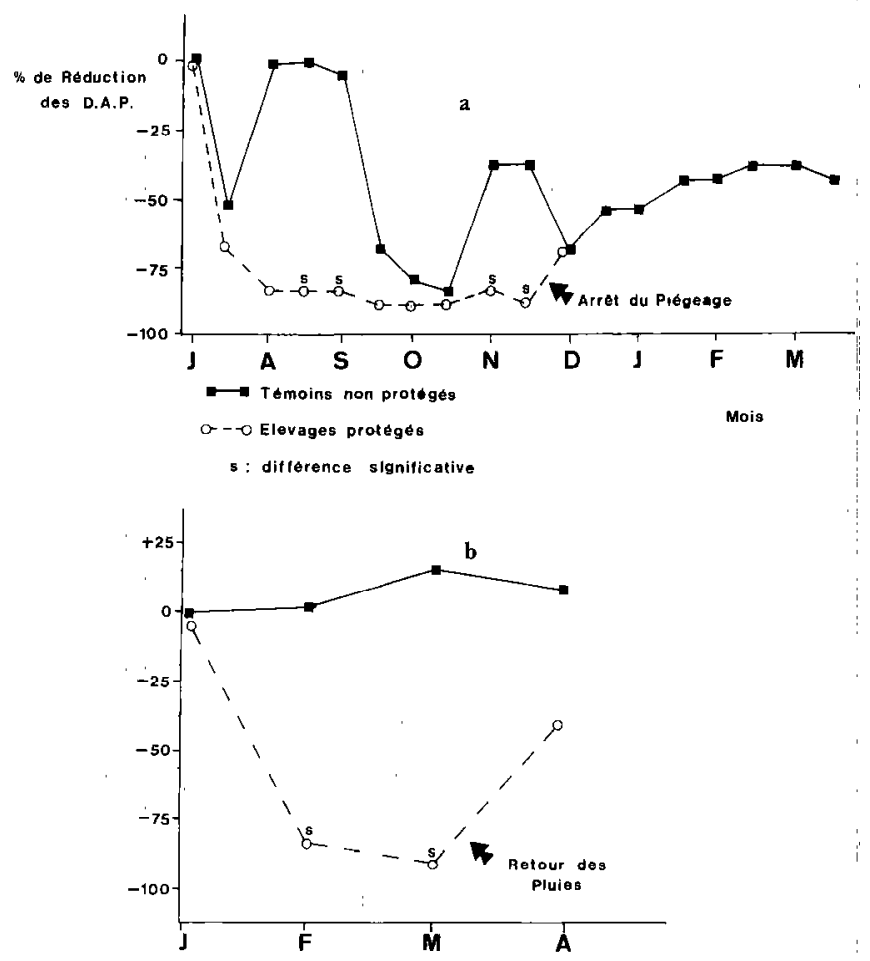

Figure 3 : Différences des DAP de G. f. fuscipes entre abreuvoirs piégés (P) et non piégés (NP) des campements de saison des pluies (a) et de saison sèche (b). Moyennes des pourcentages de réduction des DAP sur 19, sites.

aux abreuvoirs des lieux de transhumance, jusqu'en: avril). Cette réduction est de 85 p. 100 par rapport aux DAP initiales, en accord avec des résultats antérieurs en RCA (7). La courbe sur les abreuvoirs NP (fig. 3a) indique une pullulation relative des glossines en saison des pluies déjà signalée $(6,8)$. Des variations importantes des DAP sont enregistrées en saison des pluies. aux abreuvoirs NP.

Une remontée des captures aux abreuvoirs $P$ de saison! des pluies désertés se produit dès l'arrêt du piégeage, lors du départ en transhumance, jusqu'aux valeurs des! abreuvoirs NP. Le retour des pluies au mois de mars (fig. 3b) se traduit également par un accroissement relatif des DAP mesurées aux abreuvoirs $P$; cet accroissement n'apparaît pas sur les abreuvoirs NP.

\section{Parasitologie}

La prévalence annuelle moyenne (juin 1990 à fóvrier 1991) du lot NP (18 p. 100) est supérieure à celle du lot $P$ $(10,5 \mathrm{p} .100)$, tous types d'infections et d'espèces: confondus. Les prévalences comparées des lots $P$ et NP. au cours des trois premières visites, pour chaque type d'infection et pour chaque espèce ainsi que leur significa-! tivité, figurent au tableau I. 
TABleau I

Prévalences trypanosomiennes (parasitologie)

Test de comparaison des moyennes

\begin{tabular}{|c|c|c|c|c|c|c|}
\hline & \multicolumn{2}{|l|}{ Visite 1} & \multicolumn{2}{|c|}{ Visite 2} & \multicolumn{2}{|c|}{ Visite 3} \\
\hline & NP & $\mathbf{P}$ & NP & $\mathbf{P}$ & NP & $\mathbf{P}$ \\
\hline $\begin{array}{l}\text { Tb } \\
\text { Tc } \\
\text { Tv } \\
\text { Simple }\end{array}$ & $\begin{array}{c}0 \\
3,5 \\
8,7 \\
12,2\end{array}$ & $\begin{array}{l}0 \\
3 \\
6,2 \\
9,2\end{array}$ & $\begin{array}{c}0 \\
2,5 \\
17,8 \\
20,3\end{array}$ & $\begin{array}{c}0 \\
2,2 \\
9,5^{5} \\
11,7^{5}\end{array}$ & $\begin{array}{c}0 \\
1,9 \\
15,4 \\
17,3\end{array}$ & $\begin{array}{l}0 \\
2,3 \\
6,6^{\mathrm{s}} \\
8,9^{\mathrm{s}}\end{array}$ \\
\hline $\begin{array}{l}\text { TbTc } \\
\text { TbTv } \\
\text { TcTv } \\
\text { Mixte }\end{array}$ & $\begin{array}{l}0 \\
0 \\
0,9 \\
0,9\end{array}$ & $\begin{array}{l}0 \\
0,2 \\
0,2 \\
0,5\end{array}$ & $\begin{array}{l}0 \\
0 \\
3 \\
3\end{array}$ & $\begin{array}{l}0 \\
0 \\
0 \\
0\end{array}$ & $\begin{array}{l}0 \\
0 \\
0 \\
0\end{array}$ & $\begin{array}{l}0 \\
0 \\
0,7 \\
0,7\end{array}$ \\
\hline TbTcTV & 0,4 & 0 & 0 & 0 & 0 & 0 \\
\hline $\begin{array}{l}\text { Total Tb } \\
\text { Total To } \\
\text { Total TV }\end{array}$ & $\begin{array}{c}0,4 \\
48 \\
10\end{array}$ & $\begin{array}{l}0,2 \\
3,2 \\
6,7\end{array}$ & $\begin{array}{c}0 \\
5,4 \\
20,8\end{array}$ & $\begin{array}{l}0 \\
2,2^{\mathrm{s}} \\
9,5^{\mathrm{s}}\end{array}$ & $\begin{array}{c}0 \\
1,9 \\
15,4\end{array}$ & $\begin{array}{l}0 \\
3 \\
7,2^{s}\end{array}$ \\
\hline Tatal & 13,5 & 9,7 & 23,3 & $11,7^{\mathrm{s}}$ & 17,3 & $9,5^{\mathrm{s}}$ \\
\hline
\end{tabular}

Visite 1 : test bilatéral ; visites 2 et 3 : test unilatéral.

NP : troupeaux non protégés; $P$ : troupeaux protégés.

$\mathrm{Tb}$ : pourcentage d'infections à T.b. brucei; Tc : pourcentage d'infections à $T$. congolense; Tv : pourcentage d'infections à $T$. vivax.

TbTc/TbTV/TcTV : pourcentages d'infections mixtes par deux espèces de trypanosomes ; Mixte : pourcentages d'infections mixtes toutes espèces

confondues.
TbTcTv: pourcentages d'infections multiples.

TbTCTV : pourcentages d'infections multiples.
Total Tb : Fréquence d'infections à T.b. brucei décelées

Total Tc : Fréquence d'infections à T. congolense décelées ;

Total TV : Fréquence d'infections à $T$. vivax décelées.

La prévalence annuelle moyenne est, pour l'ensemble des animaux, de 13 p. 100 avec une nette prédominance des infections simples (94 p. 100) par rapport aux infections mixtes (associant deux espèces) (5,5 p. 100) et multiples (associant $T$. vivax, $T$. congolense et $T$. $b$. brucei) $(0,5 \mathrm{p} .100)$. T. vivax est l'espèce majoritaire (9,6 p. 100 en infection simple). T. b. brucei n'a pas été mis en évidence en infection simple ; il est retrouvé une fois associé à $T$. vivax et une fois en infection multiple. $T$. congolense est rencontré en infection simple $(2,6 \mathrm{p}$. $100)$; il constitue, associé à $T$. vivax, l'essentiel des infections mixtes. La fréquence pour l'ensemble des infections est de 75 p. 100 pour $T$. vivax, de 24 p. 100 pour $T$. congolense et de $1 p$. 100 pour T. b. brucei. Les variations saisonnières des prévalences indiquent pour l'ensemble des animaux une nette augmentation des prévalences en saison des pluies : 16 p. 100 contre 11 p. 100 en saison sèche.

\section{Sérologie}

Les prévalences annuelles moyennes (juin 1990 à février $1991)$ des lots NP (66 p. 100) et P (69 p. 100), tous types
I'ABLEAU II

Prévalences trypanosomiennes (sérologie). Test de comparaison des moyennes

\begin{tabular}{|c|c|c|c|c|c|c|}
\hline \multirow[t]{2}{*}{ " } & \multicolumn{2}{|c|}{ Visite 1} & \multicolumn{2}{|c|}{ Visite 2} & \multicolumn{2}{|c|}{ Visite 3} \\
\hline & NP & $\mathbf{P}$ & NP & $\mathbf{P}$ & NP & $\mathbf{P}$ \\
\hline $\begin{array}{l}\mathrm{b} \\
\mathrm{c} \\
\mathrm{v} \\
\text { simple }\end{array}$ & $\begin{array}{r}2,4 \\
9,5 \\
10,1 \\
21,9\end{array}$ & $\begin{array}{c}1,2 \\
6,9 \\
17,1^{\mathrm{s}} \\
25,2\end{array}$ & $\begin{array}{r}0,8 \\
27,3 \\
10,7 \\
38,8\end{array}$ & $\begin{array}{c}1,7 \\
14,5^{s} \\
15,7 \\
31,8\end{array}$ & $\begin{array}{c}1,3 \\
9 \\
6,4 \\
16,7\end{array}$ & $\begin{array}{c}0,6 \\
9 \\
9,7 \\
19,3\end{array}$ \\
\hline $\begin{array}{l}\text { TbTe } \\
\text { TbTy } \\
\text { TcTV } \\
\text { Mixte }\end{array}$ & $\begin{array}{l}7,7 \\
4,1 \\
7,7 \\
19,5\end{array}$ & $\begin{array}{c}8,4 \\
0,9 \\
14^{\mathrm{s}} \\
23,4\end{array}$ & $\begin{array}{r}4,1 \\
0,8 \\
6,6 \\
11,6\end{array}$ & $\begin{array}{c}2,1 \\
0 \\
26^{\mathrm{s}} \\
28,1^{\mathrm{s}}\end{array}$ & $\begin{array}{r}16 \\
1,3 \\
8,3 \\
25,6\end{array}$ & $\begin{array}{r}6,9 \\
4,4 \\
12,5 \\
23,7\end{array}$ \\
\hline hTCTy & 23,1 & $14,3^{s}$ & 5 & 9,1 & 36,5 & 31,8 \\
\hline $\begin{array}{l}\text { Total Tb } \\
\text { Total Tc } \\
\text { Total TV }\end{array}$ & $\begin{array}{l}37,3 \\
47,9 \\
45\end{array}$ & $\begin{array}{l}24,9^{s} \\
43,6 \\
46,4\end{array}$ & $\begin{array}{l}10,7 \\
43 \\
23,1\end{array}$ & $\begin{array}{l}12,8 \\
51,7 \\
50,8^{s}\end{array}$ & $\begin{array}{l}55,1 \\
69,9 \\
52,6\end{array}$ & $\begin{array}{l}43,6 \\
60,1 \\
58,3\end{array}$ \\
\hline Tota & 64,5 & 62,9 & 55,4 & $69^{5}$ & 78,8 & 74,8 \\
\hline
\end{tabular}

Visite 1 : test bilatéral ; visites 2 et 3 : test unilatéral.

NP : troupeaux non protégés ; $P$ : troupeaux protégés.

$\mathrm{Tb}$ : pourcentage d'infections à T.b. brucei; Tc : pourcentage d'infections à $T$. congolense; Tv: pourcentage d'infections à $T$. vivax.

TbTc/TbTV/TcTv: pourcentages d'infections mixtes par deux especes de trypanosomes; Mixte : pourcentages d'infections mixtes toutes espèces confondues.

TbToTv: pourcentagcs d'infections multiples.

Total Tb : Fréquence d'infections à T.b. brucei décelées

Total Tc: Fréquence d'infections à $T$. congolense décelées

Total Tv : Fréquence d'infections à $T$. vivax décelées.

d'infections et d'espèces confondus; ne sont pas significativement différentes. Les prévalences comparées des troupeaux piégés et témoins au cours des 3 premières visites, pour chaque type d'infection et pour chaque espèce ainsi que leur significativité, figurent au tableau II.

La prévalence annuelle moyenne pour l'ensemble des animaux est de 68 p. 100 ; les types d'infections sont diagnostiqués avec une fréquence à peu près identique à celles obtenues en parasitologie.' $T$. b. brucei reste l'espèce minoritaire (1,3 p. 100 en infection simple, fréquence d'apparition de 23 p. 100) ; $T$. congolense et $T$. vivax atteignent des prévalences de l'ordre de 12 p. 100 en infection simple et constituent respectivement 40 et 37 p. 100 des infections décelées. T. congolense-T. vivax est l'association la plus fréquemment rencontrée en infection mixte (59 p. 100 des infections mixtes). Les variations saisonnières des prévalences pour l'ensemble des animaux présentent presque tous les cas de figures. L'augmentation des prévalences en saison des pluies détectée en parasitologie ne se retrouve pas en sérologie ; c'est même le contraire qui se produit (65 p. 100 en saison des pluies contre 76 p. 100 en saison sèche). 
F. Le Gall F. Blanc J.P. Gouteux M. Mainguet D. Cuisance J.L. Lemesre S. Nitcheman M. Cavaleyra F. D'Amico E. Pounékrouzou F. N'Dokoué

\section{Hématocrite}

L'évolution des valeurs de l'hématocrite au cours des visites montre une différence significative entre les lots de troupeaux $P$ et NP. Les valeurs plus élevées au départ pour le lot témoin NP ne sont pas significatives. Au cours des visites les valeurs des hématocrites du lot $P$ restent stables alors que celles du lot NP baissent significativement de la première visite à la seconde et de la première visite à la troisième (fig. 4).

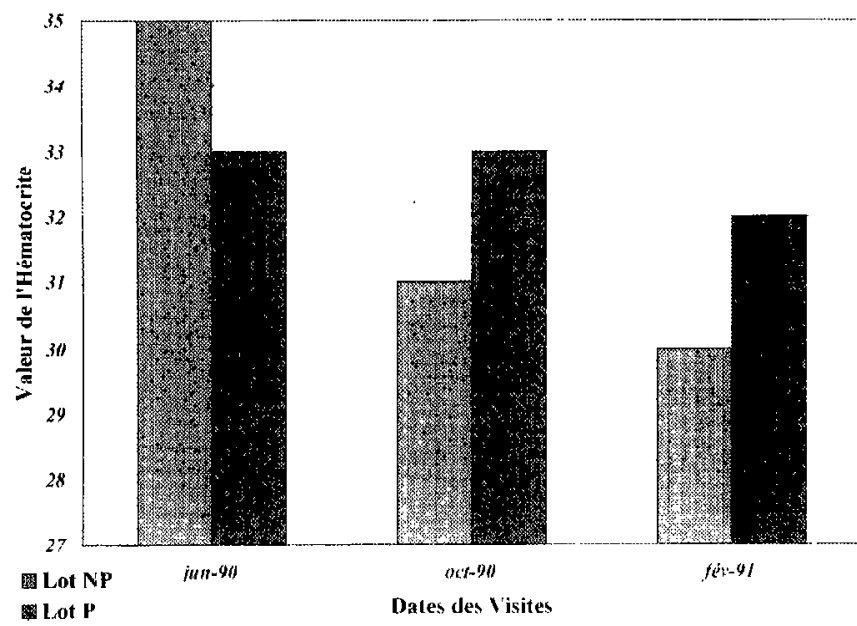

Figure 4 : Evolution comparée des valeurs de l'hématocrite.

Les valeurs des hématocrites des lots $P$ et NP ne sont pas significativement différentes à la mise en place du réseau (juin 1990). L'évolution de ces valeurs entre les visites I et 2 (octobre 1990) d'une part, et les visites 1 et 3 (février 1991) d'autre part, est significativement différente en faveur du lot $P$ (test de Mann-Whitney, comparaison unilatérale).

\section{Zootechnie}

Les paramètres de fécondité, de mortalité et de quantité de lait trait des lots $P$ et NP, calculés à partir des paramètres observés sur les animaux des ensembles $T+$ et $T$, figurent au tableau III. II n'apparaît de différence significative à l'échelle du troupeau entre les lots $P$ et NP que pour le nombre de traitements réalisés contre les trypanosomoses (index Bérénil ou "trypanocide").

\section{DISCUSSION}

\section{Suivi d'un réseau}

Un des points forts du programme est l'évaluation de la lutte en vraie grandeur et en condition d'élevage traditionnel par le suivi individuel des animaux. Le suivi simultané de plusieurs troupeaux témoins dispersés dans la zone d'étude permet de prendre en compte la grande diversité de situations entomologiques et de gestion des troupeaux à l'intérieur d'une région apparemment uniforme. Aucun travail d'évaluation d'une lutte par piégeage pour la protection de l'élevage n'avait jusqu'en 1992 rempli ces deux conditions $(17,22)$.
TABLEAU III

Paramètres zootechniques calculés pour les lots $P$ et NP

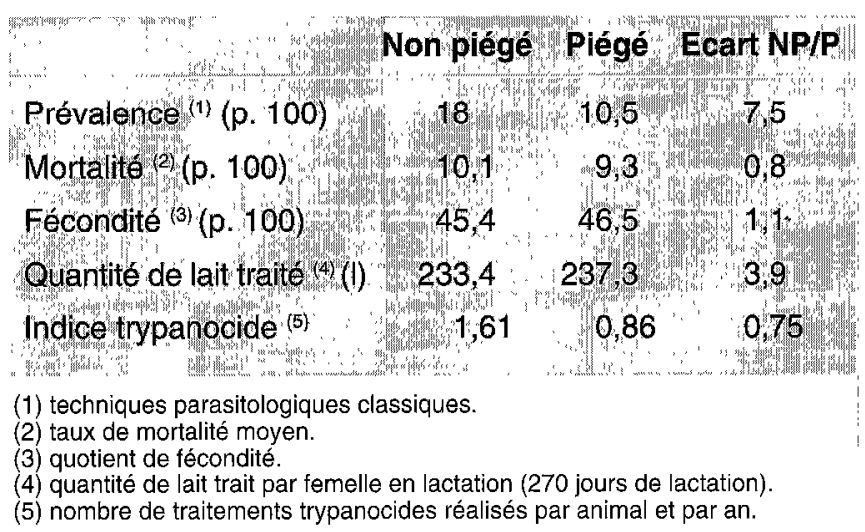

Il faut insister sur la difficulté d'un suivi individuel d'un trou-' peau en milieu Mbororo, particulièrement durant la transhumance. L'enquêteur doit être conscient de la variabilité de l'échantillon au cours des visites et critique quant aux informations recueillies ou communiquées par l'éleveur. En saison sèche les éleveurs se déplacent, changent fréquemment d'abreuvoir, et sont parfois difficilement accessibles. Le piégeage doit être continu en toute saison (pose des pièges à chaque nouvel abreuvoir) et faire l'objet d'une surveillance attentive (contre les détériorations, lesi crues...). Cette continuité de la lutte est possible en saison: des pluies, lorsque les éleveurs demeurent sur un même' campement; elle n'est efficace durant la transhumance. que si le troupeau stationne au moins 1 mois au même endroit ; les deux mois de transhumance itinérante constituent une période particulièrement défavorable où le piégeage n'est en pratique pas réalisé.

\section{Entomologie}

Peu de G. fusca congolensis ont été capturées au cours de ce suivi ; ceci confirme la régression du groupe fusca dans cette région (14). Cette régression serait liée à la colonisation accrue des savanes humides par les agriculteurs, les. pasteurs et leurs troupeaux repoussant à la fois le gros' gibier et les glossines qui lui sont inféodées.

La pullulation des glossines en saison des pluies est bien connue des éleveurs : ils ne se rendent sur les pâturages les plus infestées du Sud qu'en saison sèche, lorsque les glossines y sont les moins nombreuses, et sont forcés de s'en retirer dès le retour des pluies.

Les chutes de densités en saison des pluies enregistrées sur les abreuvoirs NP sont vraisemblablement dues à de brusques crues ; ces crues déciment temporairement les populations glossiniennes en noyant les gîtes à pupes. De telles variations peuvent également s'expliquer par les cycles de reproduction et de repas des glossines (3). La remontée rapide des DAP à l'arrêt du piégeage jusqu'aux valeurs des DAP des abreuvoirs NP n'est pas surprenante : le piégeage aux abreuvoirs est une méthode 
de contrôle qui diminue la pression glossinienne. G. $f$. fuscipes se déplace linéairement à l'intérieur des galeries et peut coloniser rapidement les abreuvoirs désertés. Cette remontée souligne l'importance d'un piégeage en continu et une certaine difficulté d'application de cette méthode dans le cas d'éleveurs transhumants.

L'impact du piégeage sur les DAP (7) et l'efficacité du piège bipyramidal Gouteux-ANDE deux fois supérieure à celle du meilleur piège utilisé jusqu'alors contre $G$. $f$. fuscipes (15) étaient connus. L'objet de ce suivi était de vérifier que la réduction des DAP entraînait une baisse des prévalences trypanosomiennes et d'en déterminer les effets sur la productivité du cheptel. L'accroissement relatif des DAP aux abreuvoirs $P$ (fig. $3 b$ ) peut être le résultat d'un biais: la fin de la saison sèche marque le départ des éleveurs vers leur campement et donc un entretien moindre de leurs pièges. La crainte que les crues n'emportent leurs pièges les pousse en outre à relever ces pièges, d'où une moindre efficacité de la lutte.

Une réduction considérable (85 p. 100) des populations glossiniennes n'est pas toujours suivie d'une baisse aussi nette des prévalences trypanosomiennes en parasitologie (23). Leak et al. (18) concluent ainsi : "the necessity in tse tse control campaigns for a major reduction of tse tse populations to take place before significant decreases in trypanosome incidence in livestock can be achieved". Les résultats trouvés ici confirment cette observation : une réduction des DAP de 85 p. 100 ne s'est traduite par une baisse des prévalences trypanosomiennes que de 18 à $10,5 p$. 100. Certes un impact a été noté lors de ce suivi ; les relations entre DAP et prévalences trypar osomiennes restent toutefois insuffisamment connues. L'intervention de vecteurs mécaniques (en particulier les stomoxes) n'est donc pas à exclure comme le suggère un travail récent $(9,10)$.

\section{Hématocrite}

La lutte par piégeage au niveau des abreuvoirs permet de maintenir les hématocrites à une valeur moyenne acceptable (> 32). La valeur de l'hématocrite, facile à mesurer sur le terrain, est considérée comme un bon critère de diagnostic $(4,5,11)$. Les chutes de l'hématocrite enregistrées sur les troupeaux NP sont un élément important en faveur de l'efficacité du piégeage, et particulièrement en faveur d'un contrôle des répercussions cliniques des trypanosomoses.

\section{Parasitologie et sérologie}

L'impact du piégeage est net en saison des pluies comme en saison sèche sur les prévalences trypanosomiennes en parasitologie et sur $T$. vivax en particulier, espèce majoritairement mise en évidence.

L'interprétation des résultats de sérologie est plus délicate : l'impact du piégeage n'apparaît en saison des pluies que pour $T$. congolense et en saison sèche que pour l'association T. b. brucei-T. congolense. Le cas de $T$. vivax est intéressant puisque les résultats de la sérologie sont inverses de ceux de la parasitologie : différence significative en défaveur du piégeage en saison des pluies et non significative en saison sèche. Puisqu'il a été mis en évidence que le piège a un impact vis-à-vis de $G$. $f$. fuscipes, vis-à-vis de $T$. vivax en parasitologie et sur les paramètres zootechniques, certains points demandent à être précisés : sensibilité et spécificité comparées des deux méthodes, mécanismes d'équilibre parasite-hôte, existence et importance d'une transmission mécanique de T.vivax (10), efficacité d'un piégeage limité aux abreuvoirs sur cette transmission mécanique (9).

Les méthodes de parasitologie classique sont peu sensibles pour la détection d'un animal trypanosomé et pour la mise en évidence des infections mixtes ou multiples. Leurs résultats sont par contre bien corrélés à l'incidence de la trypanosomose clinique. Les différences entre les deux lots, fortes pour la parasitologie, s'estompent avec la recherche d'antigènes circulants.

Pour expliquer ce fait, plusieurs hypothèses peuvent être avancées :

- il pourrait s'agir d'une trop faible spécificité (présence de faux positifs) et/ou sensibilité (pour $T$. vivax par exemple) de la technique ELISA-Ag., signalée par certains (16) ;

- les animaux protégés par les pièges sont soumis à une pression trypanosomienne moins forte et arrivent à mieux contrôler leur parasitémie. Les techniques de parasitologie détectent les animaux à forte parasitémie sur lesquels des répercussions cliniques se manifestent ; ce sont ces techniques qui reflètent le mieux l'effet protecteur du piégeage et particulièrement les bénéfices économiques de cette lutte. Si la technique ELISA-Ag. est validée (travail actuellement entrepris par l'IAEA sur un certain nombre de pays africains), elle indiquerait plus un portage qu'une affection clinique dans la mesure où elle détecte même les animaux à faible parasitémie ; il apparaît alors nécessaire d'ajouter à l'analyse qualitative (différenciation d'espèces) une analyse quantitative des degrés de parasitémie.

\section{Zootechnie}

L'amélioration des paramètres zootechniques n'a pas pu être mise en évidence directement par comparaison entre lots $P$ et NP, mais par comparaison entre animaux $T+$ et $T$ répartis dans les différents troupeaux. II est probable, au vu des résultats parasitologiques et des valeurs de l'hématocrite, qu'un suivi sur plusieurs années d'un plus grand nombre de troupeaux mette en évidence cet impact. Les résultats obtenus sur le réseau permettent néanmoins une évaluation de l'impact du piégeage sur la productivité à partir des paramètres calculés. L'analyse coût-bénéfice développée sur plusieurs années devrait établir la bonne rentabilité de la technique de lutte par piégeage ; cette analyse fera l'objet d'une publication ultérieure. 


\section{F. Le Gall F. Blanc J.P. Gouteux M. Mainguet D. Cuisance J.L. Lemesre S. Nitcheman M. Cavaleyra F. D'Amico E. Pounékrouzou F. N'Dokoué}

\section{CONCLUSION}

Cette étude sur un réseau de troupeaux confirme que les trypanosomoses bovines sont un problème majeur pour les éleveurs Mbororo en zone humide. Ces pasteurs consacrent d'ailleurs une part importante de leur budget à l'achat de trypanocides (20).

Le piégeage aux abreuvoirs permet de contrôler les populations de G. $f$. fuscipes. II maintient leur densité et le contact bétail/tsé-tsé à un niveau suffisamment bas pour diminuer les prévalences révélées sur le bétail en parasitologie, et permettre peut-être à l'animal de surmonter une trypanosomose clinique. Les animaux détectés $\mathrm{T}+$ en parasitologie correspondent aux formes cliniques ; ils présentent corrélativement une valeur faible de l'hématocrite. Les paramètres de productivité se trouvent améliorés et les achats de trypanocides diminuent consécutivement à ce contrôle vectoriel.

Ce programme a permis de tester la technique ELISA-Ag. Cette technique, si elle se révélait fiable, indiquerait une forte prévalence trypanosomienne sur le bétail des savanes humides de RCA, que les techniques de parasitologie ne reflètent pas. La spécificité et la sensibilité de la technique restent donc à évaluer. La signification respective et les rapports entre les prévalences détectées en sérologie et en parasitologie, ainsi qu'entre le portage trypanosomien et les formes cliniques, requièrent plus de précisions.

Le suivi d'un réseau en milieu réel est difficile mais d'un grand intérêt en recherche-développement car il prend en compte l'éleveur et ses pratiques. Si le piège est efficace, son acceptation et son utilisation adéquate par les bénéficiaires restent délicates. La réussite d'une lutte par piégeage à plus grande échelle repose sur la qualité des opérations de démonstration-vulgarisation devant la précéder et l'accompagner.

\section{Remerciements}

Le programme a bénéficié d'un co-financement Gouvernement centrafricain, Banque mondiale/FIDA, Fonds d'aide et de coopération (France), Fonds européen de développement (UE).

Les auteurs tiennent à remercier le Dr Kota-Guinza, Directeur général de l'ANDE, pour la bienveillante attention qu'il a accordée à nos travaux, le CIRAD-EMVT pour ses précieux conseils à la mise en place du programme, le Centre ORSTOM de Montpellier, à travers son équipe du Laboratoire d'épidémiologie des grandes endémies tropicales, qui a bien voulu procéder à l'analyse des sérums, I'ILRAD (International Laboratory for Research on Animal Diseases) de Nairobi (devenu ILRI) qui a fourni les anticorps monoclonaux, ainsi que toute l'équipe du service Entomologie et Protozoologie de la Direction de la Santé animale et de la recherche appliquée de l'ANDE.

\section{Bibliographie}

1. BLANC F., GOUTEUX J.P., CUISANCE D., POUNÉKROZOU E., LE MASSON A, N'DOKOUE F. MAINGUET M. D'AMICO F. LE GALL F., 1991. La lutte par piégcage contre Glossina fuscipes fuscipes pour la protection de l'élevage en République centrafricaine. III. Vulgarisation en milieu Mbororo. Revue Élev. Méd. vét. Pays trop., 44 (3) : 301-307.

2. BLANC F., GOUTEUX J.P., CUISANCE D., POUNÉKROZOU E., N'DOKOUÉ F., LE GALL F., 1991. Etude de la répartition des tsé tsé (Diptera : Glossinidae) en zone de savane humide (République centrafricaine). Evaluation de techniques de prospection entomologique. Trop. Med: Parasit., 42: 127-130.

3. CHALLIER A., 1973. Ecologie de Glossina palpalis gambiensis Vanderplank 1949 (Diptera-Muscidae) en savane d'Afrique Occidentale. Paris, France, ORSTOM, 274 p. (ORSTOM mémoire' ${ }^{\circ}{ }^{\circ} 64$ )

4. CONNOR R.J., 1989. Final report of the regional trypanosomosis expert. Harare, Zimbabwe, RTTCP-CEE, 123 p.

5. COULIBALY L., DIARRASOUBA L., D'IETEREN G., ITTY P., MAEHL H., MAHAMAT B., NAGDA S., PALING R., RARIEYA I., SCHUETTERLE A., THORPE W., TRAIL J., 1988. Effect of endemic diseases including trypanosomosis on the blood cell volum packed of livestock in Northern Côte d'Ivoire. Projet conjoint SODEPRA. ISCTRC, p. 552-555. (Publication No. 144)

6. CUISANCE D., 1988. Bilan de quatre missions d'appui à l'unité de lutte contre les glossines dans le cadre du projet national de développement de l'élevage. Maisons-Alfort, France, IEMVT, BDPA-SCETAGRI, 62 p.

7. CUISANCE D., CALITON P., KO'TA-GUINZA A., N'DOKOUÉ F., POUNEKKROZOU E., DEMBA D., 1991. Lutte contre Glossina fuscipes fuscipes par piégeage chez les éleveurs Mbororo de République centrafricaine. Revue Elev. Méd. vét. Pays trop., 44 (1) : 81-89.

8. CUISANCE D., GOUTEUX J.P., CAILTON P., KOTA-GUINZA A N'DOKOUÉ F., POUNÉKROZOU E., 1992. Problématique d"une lutte contre les glossines pour la protection de l'élevage en République centrafricaine. Mém. Soc. r. belge Ent., 35 : 103-110.

9. D’AMICO F., 1993. Rôle de Glossina fuscipes fuscipes Newstead, 1910 dans la transmission des trypanosomoses bovines en Afrique centrale. Cas de la zonc d'ćlevage d'Ouro-Djafoun (Rćpublique centrafricaine). Thèse doct., université Montpellier II, France, 160 p.

10. D'AMICO F., GOUTEUX J. P., LE GALL F., CUIS $\triangle$ NCE D. Are stable flies (Diptera : Stomoxyinae) vectors of Trypanosoma vivax in Central African Republic ? Accepté par Vet. Res.

11. FÉRON A., D’IETEREN G., ITTY P., MAEHL H., MULUNGO M., NAGDA S., PALING R., RARIEYA M., SHERIA M., THORPE W., TRAIL J., 1987. L'hématocrite peut-il servir d'indice de trypanosnmiase et de niveau de production chez les bovins? In: 19e Réunion CSIRLT, Lomé, Togo, 1987. Nairobi, Kenya, OUA/CSTR, p. 534-537. (Publication ${ }^{\circ}{ }^{114}$ )

12. FINELLE P., ITARD J., YVORE P., LACOTTE R., 1963. Répartition des glossines en République centrafricaine. Etat actuel des connaissances. Revue Élev. Méd. vét. Pays trop., 16 (3) : 337-348.

13. GOUTEUX J.P., 1991. La lutte par piégeage contre Glossina fuscipes fuscipes pour la protection de l'élevage en République centrafricaine. II. Caractéristiques du piège bipyramidal. Revue Elev. Méd. vét. Pays trop. 44 (3) : 295-299.

14. GOUTEUX J.P., 1992. La raréfaction de tsé-tsé du groupe fusca (Diptera : Glossinidae) en Afrique Centrale. Bull. Soc. ent. Fr., 96 (5) : 443449 .

15. GOUTEUX J.P., CUISANCE D., DEMBA D., N'DOKOUÉ F., LE GALL F., 1991. La lutte par piégeage contre Glossina fuscipes fuscipes 
pour la protection de l'élevage en République centrafricaine. I. Mise au point d'un piège adapté à un milieu d'éleveurs semi-nomades. Revue Élev. Méd. vét. Pays trop., 44 (3) : 287-294.

16. KANWE A.B., BENGALY Z., SAULNIER D., DUVALLET G., 1992. Evaluation du test de détection des antigènes circulants de trypanosomes à l'aide d'anticorps monoclonaux. Infections expérimentales ct naturcllcs. Revue Élev. Méd. vét. Pays trop., 45 (3-4) : 265-271.

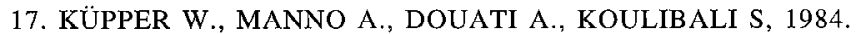
Impact des pièges biconiques imprégnés sur les populations de Glossina palpalis gambiensis et Glossina tachinoides. Résultat d'une campagne de lutte à grande échelle contre la trypanosomose animale au nord de la Côted’Ivoire. Revue Élev. Méd. vét. Pays trop., 37 (nº spécial) : 176-185.

18. I.EAK S.G.A. COLLARDEILE C., COULIBALY L., DUMONT P., FÉRON A., HECKER P., D'IETEREN G.D., JEANIN P., MINENGU M., MINJA S., MULATU W., NANKODABA G., ORDNER G., ROWLANDS G.J. SAUVEROCHE B., TIKUBET G., TRAIL J.C.M., 1990. Relationships between tsetse challenge and trypanosome prevalence in trypanotolerant and susceptible cattle. Insect Sci. Applic., 11 (3) : 293-299.

19. LE GALL F., N'DOKOUÉ F., MAINGUET M., 1992. Résultats d'une enquête large réalisée sur 27 secteurs d'élevage en RCA (1991): maladies transmises par les tiques et trypanosomoses. Espèces vectrices, coûts des mortalités et traitements. Bangui, RCA, DSARA/ANDE, 55 p. (rapport)

20. LE MASSON C., REMAYEKO A., 1990. Les éleveurs Mbororo, étude socio-économique. Bangui, République centrafricaine, Ministère du Développement rural, ANDE, $226 \mathrm{p}$

LE GALL (F), BLANC (F), GOUTEUX (J.P), MAINGUET (M.), CUISANCE (D.), LEMESRE (J.L.), NITCHEMAN (S.), CAVALEYRA (M.), D'AMICO (F.),POUNÉKROUZOU (E.), N'DOKOUÉ (F.). Control of Glossina fuscipes fuscipes by traps to protect livestock in the Central African Republic. IV. Entomological, parasitological and zootechnical impact. Revue Élev. Méd. vét. Pays trop., 1995, 48 (2) : 161-169

Neighteen Mbororo zebu herds were monitored to evaluate the impact of a trapping campaign against Glossina fuscipes fuscipes restricted to the watering places. This programme is part of the strategy of an integrated campaign against bovine trypanosomoses in the Central African Republic. Trapping reduces the densities of $G$. $f$. fuscipes and causes trypanosome prevalence to fall. These effects are shown by the improved hematocrit values and the reduced number of trypanocidal treatments administered. The impact on productivity is more difficult to assess over a short period; nevertheless, it seems clear from the parameters calculated.

Key words: Cattle - Zebu cattle - Trypanosomosis - Glossina fuscipes fisscipes - Insect control - Trap - Animal husbandry - Central African Republic.
21. MAWUENA K., 1990. Compte rendu final du projet TCP/CAF 8952 : lutte contre la typanosomiase animale. Etude de la chimiorésistance des trypanosomes aux trypanocides en République centrafricaine. Rome, Italie, FAO, $16 \mathrm{p}$.

22. MAWUENA K., YACNAMBE S., 1988. L'utilisation des pièges et écrans imprégnés d'insecticide pour la lutte contre la trypanosomose ani male. Revue Elev. Méd. vét. Pays trop., 41 (1) : 93-96.

23. MIHOK S., OTIENO L.H., TARIMO C.S., 1992. Trypanosome infection rates in tsetse flies (Diptera: Glossinidae) and cattle during tsetse control operations in the Kagera River region of Rwanda. Bull. ent. Res. 82: $361-367$

24. Ministère de la Coopération et du Développement (République française). CIRAD-EMVT, 1992. L'Herbe du Laos. Chromolaena odorata (L.) R. M. King et H. Robinson et les savanes pastorales subhumides. La plante, les effets néfastes de son extension, les moyens de la contrôler. Paris, France, Ministère de la Coopération et du Développement, CIRAD-EMVT, $16 \mathrm{p}$. (Fiches techniques d'élevage tropical n6-1992)

25. NANTULYA V.M., LINDQVIST K.J., 1989. Antigen-detection enzyme immunoassays for the diagnosis of Trypanosoma vivax, $T$. congolense and T. brucei infections in cattle. Trop. Med. Parasit., $40: 267-272$.

26. WOO P.I.K., 1970. 'The haematocrit centrituge technique for the diagnosis of African trypanosomiasis. Acta trop., $27: 384-386$.

27. YVORE P., DESROTOUR J., LAURENT J., FINELLE P., 1962. Essai d'assainissement d'une zone infestée par Glossina fuscipes fuscipes Newst. en République centrafricaine. Revue Élev. Méd. vét. Pays trop., 15 (4) : 403-410.

LE GALL (F), BLANC (F), GOUTEUX (J.P.), MAINGUET (M.) CUISANCE (D.), LEMESRE (J.L.), NITCHEMAN (S.), CAVALEYRA (M.), D'AMICO (F.),POUNÉKROUZOU (E.), N'DOKOUÉ (F.). La lucha con trampas contra Glossina fuscipes fuscipes para la protección de la ganadería en República centroafricana. IV. Impacto entomológico, parasitológico y zootécnico. Revue Élev. Méd. vét. Pays trop., 1995, 48 (2) : 161-169

Se estableció el seguimiento de una red de crianza de 19 hatos cebú Mbororo, esto con el fin de evaluar el impacto de la lucha mediante la caza con trampas contra Glossina fuscipes fuscipes, limitada a los abrevaderos. El programa se inscribe en la estrategia de la lucha integrada contra las tripanosomosis bovinas en República centroafricana. La caza con trampas permite la disminución de las densidades de $G$. $f$. fuscipes, así como de las prevalencias tripanosómicas. Los efectos se traducen por una mejoría en los valores del hematocrito y por una reducción de la cantidad de tratamientos tripanocidas efectuados. El impacto sobre las productividades es más difícil de demostrar a corto plazo, sin embargo este parece manifestarse sobre los parámetros calculados.

Palabras clave : Bovino - Cebú - Tripanosomosis - Glossina fuscipes fuscipes - Control de insectos - Trampa - Ganadería - República centroafricana. 